\title{
Bioinspired Wettability-Controlled Surfaces with Gradient Micro- and Nanostructures
}

\author{
Yongmei Zheng \\ Beihang University \\ Xueyuan Road37, Haidian District, Beijing, 100191, China \\ zhengym@buaa.edu.cn
}

\section{Extended Abstract}

Biological surfaces provide endless inspiration for design and fabrication of smart materials with unique wettability. It has recently been revealed to have become a hot research area in the materials science world [1-5].

The capture silk of the cribellate spider Uloborus walckenaerius collects water through a combination of multiple gradients in a periodic spindle-knot structure after rebuilding. Inspired by the roles of micro- and nanostructures (MNs) in the water collecting ability of spider silk[2], a series of bioinspired gradient fibers has been designed by integrating fabrication methods and technologies such as dip-coating, Rayleigh instability break-up droplets, phase separation, strategies of combining electrospinning and electrospraying, and web-assembly. Through such fabrications, "spindleknot/joint" structures can be tailored to demonstrate the mechanism of multiple gradients (e.g., roughness, smooth, temperature-respond, photo-triggering, etc.,) in driving tiny water drops. A water capturing ability can be developed by the combination of "slope" and "curvature" effects on spindle-knots on bioinspired fiber. The heterostructured fibers have been fabricated by electrohydrodynamic strategies, are intelligently responding to environmental humidity. A temperatureresponsive fiber can realize the directional transport of droplet effectively. The multi-geometric gradient fiber achieves the droplet target transport in a long range along as-designed bioinspired gradient fiber. In contrast, biological surfaces such as plant leaves and butterfly wings with gradient structure features display the effect of water repellency. Smart bioinspired surfaces can be fabricated by combining machining, electrospinning, soft lithography, and nanotechnology. The gradient surfaces exhibit robust transport and controlling of droplets.

These bioinspired wettability-controlled surfaces open promising applications into anti-icing, liquid transport, antifogging/self-cleaning, water harvesting, etc.

\section{References}

[1] Y. Zheng, "Bioinspired wettability surfaces: Development in micro- and nanostructures," Pan Stanford Publishing, USA press, pp. 0-216, 2015.

[2] Y. Zheng et al, "Directional water collection on wetted spider silk," Nature, vol. 463, pp.640-643, 2010.

[3] H. Bai, L. Wang, J. Ju, R. Sun L. Jiang, Y. Zheng, "Efficient Water Collection on Integrative Bioinspired Surfaces with Star-Shaped Wettability Patterns,” Adv. Mater., vol. 26, pp. 5025-5030, 2014.

[4] M. Zhang, L. Wang, Y. Hou, S. Feng, Y. Zheng, "Controlled smart anisotropy unidirectional spreading of droplet on fibrous surface," Adv. Mater., vol. 27, pp.5057-5062, 2015.

[5] L. Wang, Q. Gong, S, Zhan, L. Jiang, Y. Zheng, "Robust Anti-Icing Performance of Flexible Superhydrophobic Surface," Adv. Mater., vol. 28, pp. 7729-7735 2016. 